\title{
Cost-utility analysis of chemotherapy in symptomatic advanced nonsmall cell lung
} cancer

\author{
C.A. Dooms*, Y.N. Lievens ${ }^{\#}$ and J.F. Vansteenkiste*
}

ABSTRACT: When using chemotherapy in patients with a short life expectancy, outcomes such as symptom improvement or clinical benefit receive increasing attention. Outcomes of subjective benefit to the patient can be rated as a utility in order to perform health economic analyses and comparisons with other treatment conditions. A cost-utility analysis has been performed alongside a prospective randomised clinical trial comparing single agent gemcitabine to cisplatin-based chemotherapy in symptomatic advanced nonsmall cell lung cancer patients.

Global quality of life as well as resource utilisation data were collected during first-line chemotherapy for both treatment arms. Incremental costs, utilities and cost-utility ratio were calculated.

Per patient, an incremental cost of $€ 1,522$ was obtained for gemcitabine compared to cisplatinvindesine, mainly as a consequence of the direct cost of the cytotoxic drugs. When combined with utilities, this resulted in an incremental cost-utility ratio for gemcitabine of $€ 13,836$ per qualityadjusted life year gained.

In conclusion, although the least expensive strategy is cisplatin-vindesine, the greater clinical benefit of gemcitabine, resulting in an acceptable incremental cost-utility ratio as compared with other healthcare interventions, balances its higher cost. The gains in subjective outcome achieved with palliative chemotherapy are critical from both a clinical and a health economic point of view.

KEYWORDS: Cisplatin-based chemotherapy, clinical benefit, cost-utility ratio, economic analysis, gemcitabine, nonsmall cell lung cancer

D ue to growing concern about the magnitude of healthcare costs in society, health policy analysts and managers in Western countries give these costs increasing attention. This trend feeds the interest in economic analyses in medical oncology. Through their daily practice, physicians have a significant impact on the utilisation of resources and on the cost of healthcare.

Lung cancer is the leading cause of cancer death in both Europe and North America [1]. The majority of these patients are diagnosed with locally advanced or metastatic disease nonsmall cell lung cancer (NSCLC). Chemotherapy is given with palliative intent and provides a modest gain in survival. The survival differences between different interventions are small. Consequently, there is an increasing interest in this palliative setting in subjective effectiveness parameters, such as symptom improvement, clinical benefit $(\mathrm{CB})$, and improvement in quality of life (QoL), which also capture differences in toxicity profile and adverse events. It is therefore equally important to consider these subjective effectiveness parameters when performing economic analyses in oncology, in order to understand how this might optimally guide decision-making.

This article reports on a cost-utility analysis (CUA) performed on the data set of a prospective randomised study comparing single agent gemcitabine to second-generation cisplatin-based chemotherapy in symptomatic advanced NSCLC.

\section{PATIENTS AND METHODS}

\section{Study database}

The data set from a prospective randomised phase III trial in symptomatic advanced NSCLC comparing single agent gemcitabine to cisplatinbased doublet chemotherapy was used. The details of the study have been described previously [2]. Briefly, between May 1996 and April 1999, 169 patients with advanced NSCLC with

\section{AFFILIATIONS}

${ }^{*}$ Respiratory Oncology Unit (Pulmonology) and Leuven Lung Cancer Group, and

\#Dept of Radiation Oncology and Leuven Lung Cancer Group, Catholic University, Leuven, Belgium.

CORRESPONDENCE

C.A. Dooms

Respiratory Oncology Unit (Dept of Pulmonology) University Hospital Gasthuisberg Herestraat 49 B-3000 Leuven Belgium Fax: 3216346803 E-mail: christophe.dooms@ uz.kuleuven.ac.be

Received:

September 012005 Accepted after revision: January 172006 
Karnofsky performance status $\geqslant 60 \%$ were randomised to either single agent therapy with the "new drug" gemcitabine or cisplatin-vindesine, the most commonly used regimen in Belgium at that time. To be eligible for the study, patients had to be symptomatic, defined as having a visual analogue score (VAS) of $\geqslant 20$ out of 100 for at least one symptom. The primary end-point of the trial was a $\mathrm{CB}$ response.

A detailed prospective follow-up of the patients has been recorded from the time of randomisation until the end of the administration of the study chemotherapy. Within this period, outcome was scored (CB, QoL and survival) and data on resource utilisation were collected prospectively. Further events in patients with disease progression (salvage therapies, such as second-line chemotherapy, far from common at that time, or palliative radiotherapy) were not recorded in detail, just their occurrence was recorded. Survival data were collected until death.

\section{Cost-effectiveness and cost-utility evaluation}

The outcome data on CB response and survival were collected for all patients enrolled in the described prospective multicentre randomised trial [2]. Patients were enrolled at different institutions throughout Belgium, both academic and general hospitals.

\section{Survival and cost-effectiveness}

In the study, no significant difference in survival was observed between the two treatment regimens. Therefore, a costeffectiveness analysis, comparing the cost of an intervention to a clinically relevant objective outcome (life years saved (LYS) being the most commonly used), was judged not to be useful. Only costs would have to be taken into account and the cisplatin-vindesine doublet would, as such, become the optimal treatment option.

\section{Quality of life, cost-utility and cost-utility ratio}

As there were significant differences in $\mathrm{CB}$ and QoL between the two treatment regimens, a CUA, relating the cost of both interventions to their gain in QoL (expressed in qualityadjusted life years (QALYs)), was performed.

To obtain values for the QoL of the patients within the study, the "How would you rate the quality of your life today?" item from the Lung Cancer Symptom Score (LCSS) QoL instrument was measured on a VAS. During the study period, these VAS scores were obtained weekly, directly from the patient and measured on a continuous scale from 0 to 100 . This VAS scoring was considered to be a reasonable alternative to the VAS "thermometer" score in the Euro-QoL EQ-5D instrument.

For the economic analysis, the VAS scores were transformed into a corresponding utility and expressed as a value between 0.00 and 1.00 . In the last step, these utilities were combined with the survival data from the randomised study in order to obtain QALYs. For the baseline calculation, the average utilities were multiplied by the LYS, an approach also used by BERTHELOT et al. [3]

For the sensitivity analyses, the QALYs were calculated from the area under the utility curve for the 24-week treatment period by making a summation of the utility per week.

\section{Cost calculation}

Resource cost data were calculated from the actual data of the prospective clinical study. Costs per patient were obtained for each regimen. Although the study was multicentric, unit costs of the Leuven University Hospital (Leuven, Belgium) were used in the baseline calculation.

The costs of the diagnostic procedures and the expenditure at the end of the treatment period were not available. However, it was assumed that these do not substantially differ between the two treatment arms.

A societal perspective was used. All costs of the study period were expressed in Euros and adjusted to the year 2000 price level. Discounting was not addressed, as both mean and median survival was $<12$ months.

\section{Direct medical costs}

These include all costs for cytotoxic drugs and concomitant medications, outpatient admissions and in-patient hospitalisations during the chemotherapy period.

The resource costs of the pharmaceuticals were obtained from the Leuven University Hospital pharmacy. Cytotoxic drug costs were calculated from the number of vials needed to provide the acquired dose for each administration during the treatment course.

Whether chemotherapy was delivered on an in- or outpatient basis was taken into account. To obtain the unit cost of hospital admission, the standard in- and outpatient "bed-day" resource cost of the Leuven University Hospital was augmented with the cost of physician wages and materials and with $€ 12.5$ per administration of chemotherapy in order to cover the cost of preparation in the pharmacy [4]. The unit costs associated with the delivery of chemotherapy are represented in table 1 . Community care costs were thought not to be significantly different in both treatment arms and therefore excluded.

\begin{tabular}{|c|c|c|}
\hline \multirow[t]{2}{*}{ TABLE } & \multicolumn{2}{|c|}{$\begin{array}{l}\text { Unit cost of cytotoxic drug and hospital } \\
\text { admission. Costs have been adjusted for } \\
\text { inflation and are based on relative costs in } 2000\end{array}$} \\
\hline & & Unit cost $€$ \\
\hline \multicolumn{3}{|c|}{ Vial cisplatin } \\
\hline $50 \mathrm{mg}$ in- & ient & 52.4 \\
\hline $50 \mathrm{mg}$ ou & tient & 59.5 \\
\hline \multicolumn{3}{|c|}{ Vial vindesine } \\
\hline $5 \mathrm{mg}$ in-p & & 104.5 \\
\hline $5 \mathrm{mg}$ outp & & 111.6 \\
\hline \multicolumn{3}{|c|}{ Vial gemcitabine } \\
\hline $1 \mathrm{~g}$ in-pat & & 131.3 \\
\hline $1 \mathrm{~g}$ outpa & & 138.4 \\
\hline $200 \mathrm{mg}$ in & atient & 28.3 \\
\hline $200 \mathrm{mg}$ o & atient & 34.5 \\
\hline \multicolumn{3}{|c|}{ Chemotherapy } \\
\hline In-patient & mission & 171.0 \\
\hline Outpatien & dmission & 154.1 \\
\hline Preparatio & by pharmacy & 12.5 \\
\hline
\end{tabular}




\section{Direct non-medical costs}

These mainly consist of travel costs. Nursing costs at home, costs of domestic help and other out-of-pocket costs were not available and not included. Again, a comparable consumption can be assumed in both treatment groups.

\section{Indirect costs}

These consist of lost wages and lost leisure time. The mean age of the present patient population (63 yrs for cisplatinvindesine; $64 \mathrm{yrs}$ for gemcitabine) suggests that the vast majority of them had already retired. Lost leisure time can be supposed to be equal in the two treatment arms. For these reasons, indirect costs were not taken into account.

\section{Costs generated after the end of the trial}

Within the trial, data on the occurrence of second-line chemotherapy and palliative radiotherapy were collected and showed no significant difference between the two treatment groups concerned. Detailed resource consumption data were not collected.

\section{Sensitivity analyses}

All costs and utilities to which this CUA was expected to be sensitive were varied within plausible ranges.

Costs were varied \pm 25 and $50 \%$. The impact of changing the cytotoxic drug cost and the in- and outpatient hospitalisation costs were evaluated separately. The effect of varying the combination of both former costs and of all treatment costs together was also analysed.

For the utilities, the area under curve (AUC) calculation represents the first type of sensitivity analysis. Mean baseline utilities varied \pm 25 and $50 \%$, as the second type of sensitivity analysis.

The sensitivity analyses were conducted on the assumption that survival benefits are not variable.

\section{RESULTS}

\section{Patients}

The original CB analysis consisted of 169 patients [2]. For the present CUA, eligible patients were considered to be those for whom the "How would you rate your quality of life today?" QoL data were available. Of these 142 patients, 69 patients received cisplatin-vindesine treatment and 73 received gemcitabine monotherapy. No separate stratification was performed for the VAS score obtained from the QoL question "How would you rate the quality of your life today?" Nevertheless, the baseline characteristics of the 142 patients enrolled in the cost analysis are reasonably well balanced across the two study arms (table 2).

\section{Objective response rate, survival and clinical benefit}

The log-rank test of the Kaplan-Meier survival curve shows no significant difference in survival between the two treatment regimens: 1-yr survival of $22.5 \%$ for cisplatin doublet and $25.4 \%$ for gemcitabine (fig. 1). A significantly larger and longer lasting CB was found for single agent gemcitabine, being most obvious in patients with objective disease stabilisation [2].

\section{Cost}

The total estimated resource cost consumed during the study period and the resulting cost per patient are shown in table 3 .

\begin{tabular}{lcc} 
TABLE 2 & $\begin{array}{l}\text { Baseline characteristics of the } \\
\text { enrolled in the cost analysis } \\
\text { Cisplatin-vindesine }\end{array}$ & Gemcitabine \\
\hline & 69 & 73 \\
Subjects n & $63(39-78)$ & $64(42-76)$ \\
Age yrs & 33 & 32 \\
Stage & 67 & 68 \\
IIIB & & 81 \\
IV & 81 & 19 \\
Sex & 19 & \\
Male & & \\
Female & & \\
\hline &
\end{tabular}

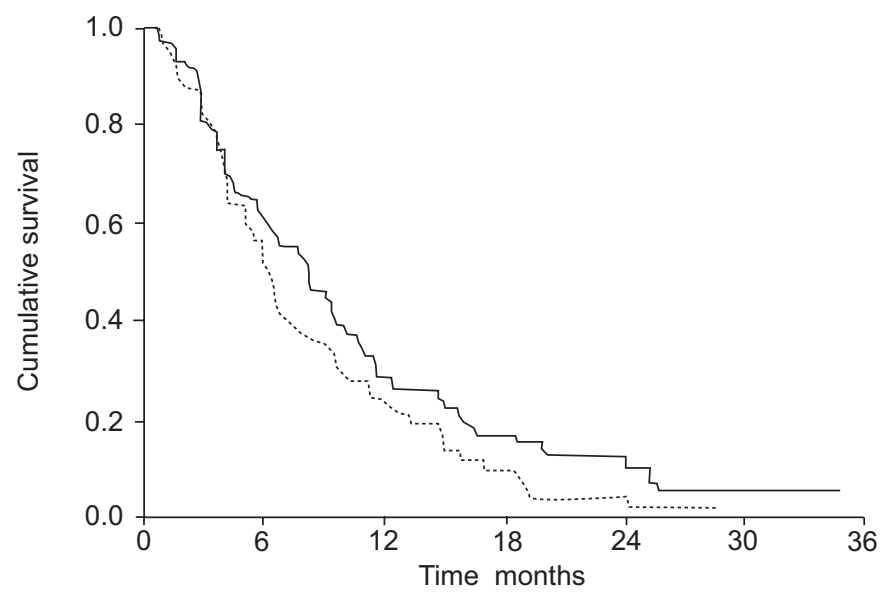

FIGURE 1. Kaplan-Meier survival curve of 142 patients enrolled in the cost analysis, according to treatment arm. ——: gemcitabine; $\cdots \cdots \cdots \cdots \cdot$. cisplatinvindesine. Log-rank $p=0.10$

\section{Utility and quality-adjusted life years}

Of all the VAS measurements, $82.5 \%$ were available. A significantly larger number of patients treated with gemcitabine had a better VAS score improvement for the global QoL question compared to cisplatin-vindesine $(37 \%$ versus $15 \%, p=0.0082)$ [5].

The baseline utility, derived from the QoL, was thus valued at 0.39 on a scale from 0.00 to 1.00 . The utility improves during the gemcitabine treatment arm and declines after four cycles of cisplatin-based therapy (fig. 2). Moreover, a generally more toxic effect of cisplatin can be suggested as a negative impact on the utility, occurring in the 3 weeks after a cisplatin administration, with recuperation during the week before a next administration (indicated by the arrows in fig. 2).

The baseline calculation showed a gain of 0.18 QALYs in the doublet arm and of 0.29 QALYs for gemcitabine monotherapy.

\section{Cost-utility ratio}

The cost-utility results for the baseline calculation are summarised in table 4 . The incremental cost of gemcitabine is $€ 1,522$ per patient and the incremental QALYs are 0.11 , resulting in a cost-utility ratio (CUR) of $€ 13,836 /$ QALYs. 


\begin{tabular}{lcc} 
TABLE 3 & $\begin{array}{c}\text { Costs associated with chemotherapy. Costs are } \\
\text { in Euros, have been adjusted for inflation and are } \\
\text { based on relative costs in } 2000\end{array}$ \\
& Cisplatin-vindesine & Gemcitabine \\
\hline & & \\
\hline & & \\
Direct medical costs & 60235 & 9080 \\
Cytotoxic drug for in-patient & 27139 & 205272 \\
Cytotoxic drug for outpatient & 85259 & 22955 \\
Hospital admission for drug delivery & 29734 & 122507 \\
Day clinic admission for drug delivery & 55188 & 50233 \\
Hospital admission for adverse event & 13697 & 5479 \\
Antibiotics & 11541 & 4021 \\
Painkillers & 16134 & 1098 \\
Anti-emetics & 2700 & 2945 \\
Transfusion & 5625 & 9963 \\
Pharmacy preparation & & \\
Direct non-medical costs & 3375 & 6188 \\
Cost of travel & & \\
Total cost & 310627 & 439739 \\
All patients & 4502 & 6024 \\
Per patient & & \\
\hline & & \\
\hline
\end{tabular}

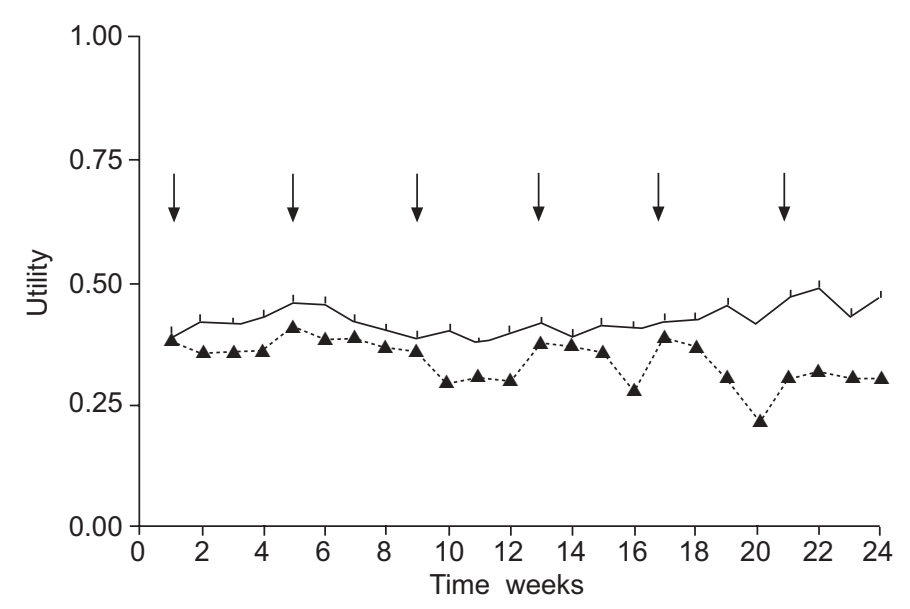

FIGURE 2. Evolution of utility for gemcitabine $(-)$ and cisplatin-vindesine ( - - - ) chemotherapy during study treatment, derived from the "How would you rate the quality of your life today?" item. Arrows indicate the administration of chemotherapy.

\section{Sensitivity analyses}

Table 5 shows how the CUR varies when applying the utilities calculated with the AUC approach. With unchanged costs, the CUR increases to $€ 38,050 /$ QALY. Furthermore, the CUR obviously follows the change in total costs. If the latter increase by 25 or $50 \%$, the CUR correspondingly increases up to a value close to and even over $€ 50,000 /$ QALY.

Table 6 shows in more detail how the results vary with different changes in cost inputs. It is notable that the cost of administration has no major impact. The cost of the cytotoxic drug, however, seems to be the most important factor influencing the CUR in the sensitivity analysis. It is still clear

\begin{tabular}{lcc} 
TABLE 4 & $\begin{array}{c}\text { Baseline cost-utility ratio. Costs are in Euros, } \\
\text { have been adjusted for inflation and are based } \\
\text { on relative costs in } 2000\end{array}$ \\
& $\begin{array}{l}\text { Cisplatin-vindesine } \\
\text { Gemcitabine }\end{array}$ \\
\hline Subjects $\mathbf{n}$ & 69 & 73 \\
Total cost & 310627 & 439739 \\
Total cost per patient & 4502 & 6024 \\
Incremental cost per patient & & 1522 \\
Median survival months & 6.3 & 8.2 \\
LYS & 0.53 & 0.68 \\
Average utility over 24 weeks & 0.34 & 0.42 \\
QALY\# & 0.18 & 0.29 \\
Incremental QALY & & 0.11 \\
CUR & & 13836 \\
\hline & & \\
LYS: life years saved; QALY: quality-adjusted life years; CUR: cost-utility ratio. \\
\#: calculated as LYS $\times$ utility.
\end{tabular}

\begin{tabular}{|c|c|c|c|}
\hline \multirow[t]{3}{*}{ TABLE 5} & \multicolumn{3}{|c|}{$\begin{array}{l}\text { Sensitivity analysis: impact of utilities calculated } \\
\text { with the area under curve (AUC) approach and } \\
\text { variation in total costs. Costs are in Euros, have } \\
\text { been adjusted for inflation and are based on } \\
\text { relative costs in } 2000\end{array}$} \\
\hline & \multirow[t]{2}{*}{ Incremental cost } & \multicolumn{2}{|c|}{ Incremental QALY } \\
\hline & & 0.11 & $0.04^{\#}$ \\
\hline All costs $-50 \%$ & 761 & 6918 & 19025 \\
\hline All costs $-25 \%$ & 1142 & 10382 & 28550 \\
\hline All costs & 1522 & 13836 & 38050 \\
\hline All costs $+25 \%$ & 1902 & 17291 & 47550 \\
\hline All costs $+50 \%$ & 2283 & 20755 & 57075 \\
\hline
\end{tabular}

from this table that even if the absolute differences in utilities remain small, the impact on the resulting CUR is important.

\section{DISCUSSION}

The role of chemotherapy in advanced NSCLC has been better defined since the publication of a meta-analysis in 1995 [6]. In the last decade, third-generation cytotoxic agents have been introduced showing almost equal activity in terms of survival but with a milder toxicity profile compared to platinum doublets $[2,7,8]$. Because of their higher cost, however, the economic consequences of introducing these new agents into the palliative setting should also be considered.

This study reports a CUA, based on data obtained from a randomised trial comparing gemcitabine monotherapy and cisplatin-vindesine for the treatment of symptomatic advanced NSCLC. A previous multicentre randomised trial showed similar efficacy in terms of survival for both regimens but pointed at a significantly better $\mathrm{CB}$ response rate in favour of gemcitabine [2]. The authors therefore examined whether the 
TABLE 6 Sensitivity analysis: impact of different cost inputs. Costs are in Euros, have been adjusted for inflation and are based on relative costs in 2000

\begin{tabular}{|c|c|c|c|c|c|c|}
\hline & Incremental cost & $\begin{array}{l}\text { Utility }-50 \% \\
\text { QALY } 0.05\end{array}$ & $\begin{array}{l}\text { Utility }-25 \% \\
\text { QALY } 0.08\end{array}$ & $\begin{array}{c}\text { Incremental utility } \\
\text { QALY } 0.11\end{array}$ & $\begin{array}{c}\text { Utility }+25 \% \\
\text { QALY } 0.13\end{array}$ & $\begin{array}{c}\text { Utility }+50 \% \\
\text { QALY } 0.16\end{array}$ \\
\hline All costs $-25 \%$ & 1142 & 22840 & 14275 & 1382 & 8785 & 7138 \\
\hline All costs & 1522 & 30440 & 19025 & 13836 & 11708 & 9513 \\
\hline All costs $+25 \%$ & 1902 & 38040 & 23775 & 17291 & 14631 & 11888 \\
\hline Drug cost $-25 \%$ & 1104 & 22080 & 13800 & 10036 & 8492 & 6900 \\
\hline All costs & 1522 & 30440 & 19025 & 13836 & 11708 & 9513 \\
\hline Drug cost $+25 \%$ & 1939 & 38780 & 24238 & 17627 & 14915 & 12119 \\
\hline Drug cost $+\mathbf{5 0} \%$ & 2326 & 46520 & 29075 & 21145 & 17892 & 14538 \\
\hline Adm cost $-50 \%$ & 1359 & 27180 & 16988 & 12355 & 10453 & 8494 \\
\hline Adm cost $+50 \%$ & 1685 & 33700 & 21063 & 15318 & 12962 & 10531 \\
\hline Drug+adm cost $-50 \%$ & 524 & 10480 & 6550 & 4764 & 4031 & 3275 \\
\hline Drug+adm cost $-25 \%$ & 1023 & 20460 & 12788 & 9300 & 7869 & 6394 \\
\hline All costs & 1522 & 30440 & 19025 & 13836 & 11708 & 9513 \\
\hline Drug+adm cost $+25 \%$ & 2021 & 40420 & 25263 & 18373 & 15546 & 12631 \\
\hline Drug+adm cost $+50 \%$ & 2489 & 49780 & 31113 & 22627 & 19146 & 15556 \\
\hline
\end{tabular}

Cost-utility ratio in €/quality-adjusted life years (QALY). QALY: quality-adjusted life years; adm: administration.

higher drug cost of gemcitabine could (partly) be offset by its improved QoL and thus result in an acceptable CUR. The present CUA confirmed this hypothesis.

Economic evaluations, weighing the benefits of interventions against their costs, are a recognised method of evaluating the financial implications related to new treatments. When dealing with modest survival benefits and potential treatment toxicity, subjective outcome measures become increasingly important in addition to the more frequently used objective parameters. Moreover, if QALYs (a non disease-specific outcome measure) are used, a direct comparison can be made for a broad range of interventions. For this reason, an incremental cost per QALY will be increasingly recommended as a useful measure to inform healthcare policy makers.

EARLE et al. [9] reviewed CUA in oncology between 1975 and 1997. Lung cancer accounted for only $7.5 \%$ of the published reports, and none of the CUA was based on chemotherapy for advanced-stage NSCLC. A systematic review ending in December 2000 reported 16 economic evaluations on the use of third-generation cytotoxic agents for inoperable stage III and IV NSCLC [10]. All types of approaches (cost-benefit analyses, cost-minimisation analyses, cost-effectiveness analyses and CUA) were evaluated in this review but only one CUA was available. This CUA of chemotherapy for metastatic NSCLC applied a decision-analysis model on a hypothetical cohort of lung cancer patients. The analysis used mean utility estimates provided by a survey amongst oncologists [3]. No other CUA alongside cancer clinical trials has as yet been reported on chemotherapy for advanced-stage NSCLC, which is probably related to the fact that the reported differences in QoL between platinum doublets only show discrete differences at best [11-15].

TennVAll and Fernberg [16] previously performed a costminimisation analysis of gemcitabine compared with cisplatinetoposide for stage IIIB-IV NSCLC. In their analysis, gemcitabine proved to be cost saving, assuming the survival and response rates in that study were equivalent. A weakness of the analysis was that no information about QoL was available. The present authors were able to perform a CUA based on patients' derived utility estimates within a comparable treatment design. Based on the standard practice of the Leuven University Hospital, an incremental societal cost of $€ 1,522$ per patient for single agent gemcitabine was calculated as compared to standard cisplatin-vindesine. After adjusting the survival time for QoL by introducing the utilities, an increment of 0.11 QALY was obtained, translating into a baseline incremental CUR of $€ 13,836 /$ QALY. This calculated incremental CUR remains below the internationally accepted benchmarks of CAN\$20,000/QALY, US\$50,000/QALY and $£ 30,000 /$ QALY used in the UK [17-19].

In the sensitivity analyses it was found that higher costs translate into higher CURs. However, in contrast to what is frequently observed, the CURs were not importantly altered by switching from in-patient drug administration to day 
hospitalisations. This is probably related to the fact that in the present authors' experience, the cost of 1-day hospitalisation does not differ greatly from the cost per day of in-patient care. Moreover, the actual number of admissions is higher in the gemcitabine arm than the doublet. In contrast, the cost picture is clearly dominated by the actual cost of the drugs.

The results of this analysis are thus sensitive to changes in costs but even more so to changes in utilities. When the impact on QoL is limited, the CURs raise considerably, as can be seen in the AUC approach. It is frequently seen in this kind of analyses that the difference in utility between treatments is more important than the absolute estimates and that the accurate measurement of health is essential for performing CUA $[4,18,20,21]$. In the present authors' trial, weekly LCSS assessments were performed. This is in contrast to the QoL assessments in the Southwest Oncology Group trial S9509 [22]. The monthly intervals between the cisplatin treatments together with the more dispersed QoL assessments in that trial possibly masked small inter-treatment QoL differences that the present authors observed and may explain why there was no significant QoL difference between both treatment arms. As a result, a cost-minimisation analysis was performed [23]. In contrast, the present authors' weekly LCSS assessment nicely demonstrated a decrease in QoL during the 3 weeks following cisplatin administration with recuperation during the fourth week prior to a new cycle of chemotherapy (fig. 2).

Although the present study yields interesting information regarding the relation between costs and QoL, it should be acknowledged that it also has limitations and methodological difficulties, such as the choice of the comparators, the utility instrument used, the length of follow-up of utility and costs, and the choice of perspective (healthcare versus societal) and institution (university versus general hospital).

First, it can be argued that single agent gemcitabine is not standard treatment in fit patients with advanced NSCLC, and that cisplatin combinations should be favoured due to the moderate survival benefit that is mostly observed. However, this was not the case in the present study. These cisplatin combinations are frequently not tolerated by frail and aged patients with symptomatic advanced NSCLC and comorbidity. Single agent therapy with a new drug such as gemcitabine therefore presents a valuable alternative. In addition, although it has been shown that modern doublets yield slightly superior survival outcomes compared to single agent therapy in fit patients with advanced NSCLC, it is less obvious whether doublet therapy is also superior to monotherapy with a new agent when subjective outcomes such as QoL or CB are considered [24, 25].

Secondly, the choice of QoL instrument used is not standard. However, utilities in oncology have been assessed by varying instruments and respondents. So far, no single standardised method is available for utility collection alongside trials. In this CUA, the global QoL item out of the LCSS QoL questionnaire was used and was assumed to be comparable to the VAS thermometer scoring of the Euro-QoL EQ-5D questionnaire. This assumption can be substantiated by the study of FosselLA et al. [26], which demonstrated that a global QoL assessment by either LCSS or Euro-QoL showed a similar outcome.
In addition, the Euro-QoL measurements obtained from NSCLC patients in a cross-sectional study using the 36-item Short-Form Health Survey demonstrated that it is useful in the evaluation of the CUR of NSCLC therapies [27]. However, it still remains possible that the QoL item may not cover all the different domains of QoL. Lastly, it was not feasible to obtain QoL measurements after the end of the study period.

The cost estimates used in the present analysis were derived from the standard unit costs and the standard resource use of the Leuven University Hospital. This approach may not entirely represent the healthcare point of view since it is not known to what extent academic cost data are representative for other institutions in this multicentre study. In the sensitivity analysis, the costs of the cytotoxic drugs and of the administration of these drugs (i.e. related to the hospital type) were therefore varied. It was found that the cost of cytotoxic administration has only limited impact on the ultimate CUR. Furthermore, indirect costs were not included in the analysis as the vast majority of the patients in this study were already retired. In some reports, however, up to $40 \%$ of the general patient population with advanced NSCLC did not reach the age of 65 , which might translate into different economic societal losses. In contrast, one issue that would not affect the results is the possible use of generic equivalents of the cytotoxics, because both cisplatin and gemcitabine had been approved $<15$ yrs ago in Belgium at the time of the analysis.

In conclusion, although cost alone should not be used to determine treatment choices in an individual patient, it cannot be ignored that the financial impact of chemotherapy in advanced-stage nonsmall cell lung cancer might be substantial when considering treatment options within a community. As the gains in hard outcomes are limited and improvements in quality of life and clinical benefit parameters are highly valued by patients with symptomatic advanced-stage nonsmall cell lung cancer, these parameters should be incorporated in health economy analyses. The present cost-utility analysis showed that the incremental cost of a third-generation cytotoxic drug (mainly related to the cost of the cytotoxic itself) is partly offset by an increased gain in quality-adjusted life years, resulting in an acceptable cost-utility ratio. However, as quality-of-life outcomes remain difficult to measure and are a critical element in determining the results of such analyses, further research is warranted in this field.

\section{REFERENCES}

1 Parker SL, Tong T, Bolden S, Wingo PA. Cancer statistics 1997. CA Cancer J Clin 1997; 47: 5-27.

2 Vansteenkiste JF, Vandebroek JE, Nackaerts KL, et al. Clinical benefit response in advanced non-small cell lung cancer. A multicenter prospective randomized phase III study of single agent gemcitabine versus cisplatinvindesine. Ann Oncol 2001; 12: 1221-1230.

3 Berthelot JM, Will BP, Evans WK, Coyle D, Earle CC, Bordeleau L. Decision framework for chemotherapeutic interventions for metastatic non-small cell lung cancer. $J$ Natl Cancer Inst 2000; 92: 1321-1329.

4 Lievens Y, Kesteloot K, Bogaert WV. CHART in lung cancer: Economic evaluation and incentives for implementation. Radiother Oncol 2005; 75: 171-178. 
5 Vansteenkiste J, Vandebroek J, Nackaerts K, et al. Influence of cisplatin-use, age, performance status and duration of chemotherapy on symptom control in advanced non-small cell lung cancer: Analysis of a randomised study. Lung Cancer 2003; 40: 191-199.

6 Non-small Cell Lung Cancer Collaborative Group. Chemotherapy in non-small cell lung cancer. A meta-analysis using updated data on individual patients from 52 randomised clinical trials. BMJ 1995; 311: 899-909.

7 Ten Bokkel Huinink WW, Bergman B, Chemaissani A, et al. Single-agent gemcitabine: an active and better tolerated alternative to standard cisplatin-based chemotherapy in locally advanced or metastatic non-small cell lung cancer. Lung Cancer 1999; 26: 85-94.

8 Perng RP, Chen YM, Ming Liu J, et al. Gemcitabine versus the combination of cisplatin and etoposide in patients with inoperable non-small-cell lung cancer in a phase II randomized study. J Clin Oncol 1997; 15: 2097-2102.

9 Earle CC, Chapman RH, Baker CS, et al. Systematic overview of cost-utility assessments in oncology. J Clin Oncol 2001; 18: 3302-3317.

10 Clegg A, Scott DA, Sidhu M, Hewitson P, Waugh N. A rapid and systematic review of the clinical effectiveness and cost-effectiveness of paclitaxel, docetaxel, gemcitabine and vinorelbine in non-small cell lung cancer. Health Technol Assess 2001; 5: 1-195.

11 Crino L, Scagliotti GV, Ricci S, et al. Gemcitabine and cisplatin versus mitomycin, ifosfamide, and cisplatin in advanced non-small-cell lung cancer: A randomized phase III study of the Italian Lung Cancer Project. J Clin Oncol 1999; 17: 3522-3530.

12 Bonomi P, Kim K, Fairclough D, et al. Comparison of survival and quality of life in advanced non-small cell lung cancer patients treated with two dose levels of paclitaxel combined with cisplatin versus etoposide with cisplatin: results of an Eastern Cooperative Oncology Group trial. I Clin Oncol 2000; 18: 623-631.

13 Cardenal F, Lopez Cabrerizo MP, Anton A, et al. Randomized phase III study of gemcitabine-cisplatin versus etoposide-cisplatin in the treatment of locally advanced or metastatic non-small cell lung cancer. J Clin Oncol 1999; 17: 12-18.

14 Giaccone G, Splinter TA, Debruyne C, et al. Randomized study of paclitaxel-cisplatin versus cisplatin-teniposide in patients with advanced non-small-cell lung cancer. The European Organization for Research and Treatment of Cancer Lung Cancer Cooperative Group. J Clin Oncol 1998; 16: $2133-2141$.
15 Evans WK, Coyle D, Gafni A, Walker H. Which cancer clinical trials should be considered for economic evaluation? Selection criteria from the National Cancer Institute of Canada's working group on economic analysis. Chronic Dis Can 2003; 24: 102-107.

16 Tennvall GR, Fernberg JO. Economic evaluation of gemcitabine single agent therapy compared with standard treatment in stage IIIB and IV non-small cell lung cancer. Med Oncol 1998; 15: 129-136.

17 Laupacis A, Feeny D, Detsky AS, Tugwell PX. How attractive does a new technology have to be to warrant adoption and utilization? Tentative guidelines for using clinical and economic evaluations. Can Med Assoc J 1992; 146: 473-481.

18 Earle CC, Coyle D, Evans WK. Cost-effectiveness analysis in oncology. Ann Oncol 1998; 9: 475-482.

19 National Institute for Clinical Excellence. Lung cancer: the diagnosis and treatment of lung cancer (clinical guideline 24). www.nice.org.uk/CG024NICEguideline. Last updated: February 2005. Last accessed: July 2005.

20 Tengs TO, Wallace A. One thousand health-related quality-of-life estimates. Med Care 2000; 38: 583-637.

21 Goodwin PJ, Shepherd FA. Economic issues in lung cancer: a review. J Clin Oncol 1998; 16: 3900-3912.

22 Moinpour CM, Lyons B, Grevstad PK, et al. Quality of life in advanced non-small cell lung cancer: results of a Southwest Oncology Group randomized trial. Qual Life Res 2002; 11: 115-126.

23 Ramsey SD, Moinpour CM, Lovato LC, et al. Economic analysis of vinorelbine plus cisplatin versus paclitaxel plus carboplatin for advanced non-small cell lung cancer. J Natl Cancer Inst 2002; 94: 291-297.

24 Dooms CA, Vansteenkiste JF. Importance of symptom management and attention to quality of life or clinical benefit of therapy in patients with advanced non-small cell lung cancer. Am J Cancer 2004; 3: 281-290.

25 Lilenbaum RC, Herndon JE, List MA, et al. Single-agent versus combination chemotherapy in advanced non-small cell lung cancer: the Cancer and Leukemia Group B study 9730. J Clin Oncol 2005; 23: 190-196.

26 Fossella F, Pereira JR, Von Pawel J, et al. Randomized, multinational, phase III study of docetaxel plus platinum combinations versus vinorelbine plus cisplatin for advanced non-small cell lung cancer: the TAX 326 study group. J Clin Oncol 2003; 21: 3016-3024.

27 Trippoli S, Vaiani M, Lucioni C, Messori A. Quality of life and utility in patients with non-small cell lung cancer. Quality-of-life study group of the master 2 project in pharmacoeconomics. Pharmacoeconomics 2001; 19: 855-863. 